\title{
Biotechnological Production Process and Life Cycle Assessment of Graphene
}

\author{
P. Noorunnisa Khanam, ${ }^{1}$ Anton Popelka, ${ }^{1}$ Maryam Alejji, ${ }^{1}$ and M. A. AlMaadeed ${ }^{1,2}$ \\ ${ }^{1}$ Center for Advanced Materials, Qatar University, 2713 Doha, Qatar \\ ${ }^{2}$ Material Science and Technology Program, Qatar University, 2713 Doha, Qatar \\ Correspondence should be addressed to M. A. AlMaadeed; m.alali@qu.edu.qa
}

Received 2 November 2016; Revised 4 December 2016; Accepted 13 December 2016; Published 12 January 2017

Academic Editor: Xiaohang Liu

Copyright (c) 2017 P. Noorunnisa Khanam et al. This is an open access article distributed under the Creative Commons Attribution License, which permits unrestricted use, distribution, and reproduction in any medium, provided the original work is properly cited.

\begin{abstract}
The aim of this study is to compare the graphene produced using a biotechnological method (Escherichia coli) with the graphene produced by Hummers' method (a chemical method) and to study the effect on the energy consumption and environment. The results indicated that the chemical reduction process has higher energy consumption, approximately $1642 \mathrm{Wh}$, than the energy consumption of the biotechnological reduction process, which is $5 \mathrm{Wh}$. The potential of global warming (GWP 100) improved by $71 \%$ using the biotechnological route for the production of graphene. Abiotic depletion, the photochemical ozone creation potential, and marine aquatic ecotoxicity potential were improved when the biological route was employed, compared with the chemical route. The eutrophication potential, terrestrial ecotoxicity, and ozone depletion layer changed very little since the main variables involved in the production of graphene oxide and waste management are the same. The biotechnological method can be considered a green technique for the production of graphene, especially given the reduction in the negative effects on global warming, abiotic depletion, the photochemical ozone creation potential, and the marine aquatic ecotoxicity potential.
\end{abstract}

\section{Introduction}

Graphene, which is a two-dimensional, single-layer sheet of $\mathrm{sp}^{2}$ hybridized carbon atoms, is very important for technology due to its excellent physical and chemical properties, including its electronic conductivity, thermal stability, and very good mechanical characteristics [1]. Graphene-related materials, which are available in other forms including graphene oxide (GO), reduced graphene oxide ( $\mathrm{rGO}$ ), and exfoliated graphite, have been used in many applications. Their excellent characteristics, ease of processability, and ready functionalization have increased the applications related to these materials, including use in electronics, photonic devices, clean energy, and sensors [2].

There are also increasing uses of graphene in bioscience and biotechnology; for instance, the use of graphene in biosensors $[3,4]$ was reported in the literature. The mass production of these materials is highly needed in modern society. To convert GO to graphene, chemical oxidation and subsequent exfoliation of $\mathrm{GO}$ are used as a common method $[5,6]$. However, the chemical reduction of GO can involve toxic chemicals and harmful vapors [7]. Thermal reduction is another method for reducing GO, but it is very complex because of the high energy used in this process. Environmental or economic concerns become critical when these two approaches are used for production of graphene on a large scale [7].

Recent reports showed that $\mathrm{GO}$ acts as a terminal electron acceptor for bacterial organisms, in which the reduction of GO is made possible by microbial effects in the breathing process or during electron transport (the electron transfer process between bacteria and GO) [6]. Microbial reduction of GO offers an alternative way to produce graphene that is rapid, cheap, and environmentally friendly [8]. The effects of graphene preparation methods on the environment can be studied by the life cycle assessment (LCA) method. The LCA method is used to measure the environmental impacts, identify the products, and also compare the impacts of different processes. Environmental assessment of nanomaterials is conducted using LCA and some of the LCA reports are 
available in the literature for other carbon nanomaterials $[9,10]$. An environmental perspective comparison of the two graphene production routes, (1) chemical reduction and (2) ultrasonication, was performed by Arvidsson et al. [9]. They concluded that the energy use and blue water footprint were approximately doubled using the chemical reduction route compared to ultrasonication, while the human toxicity of ultrasonication had the largest impact. Both routes have the same ecotoxicity impact.

In this paper, a comparison of the following two lab scale methods used for graphene preparation is presented: (1) the chemical reduction method and (2) the biotechnological reduction by Escherichia coli (E. coli). These two methods are applied to produce graphene via the reduction of GO. The comparison takes the following factors into consideration: (1) the properties and (2) the environmental impact of the two methods.

\section{Experimental}

2.1. Materials. Graphite powder (50-micron size), sulfuric acid, potassium permanganate, hydrogen peroxide, and sodium nitrite were purchased from Sigma-Aldrich for the synthesis of graphene. Distilled water was used throughout the experiment. The microorganism strain of E. coli (ATCC 11632) was used for the biotechnological reduction method of GO.

\subsection{Methods}

2.2.1. Preparation of Graphene Oxide (GO). GO was prepared from natural graphite flakes using modified Hummers' method [9]. In brief, graphite ( $3.0 \mathrm{~g})$ was added under stirring at room temperature to concentrated $\mathrm{H}_{2} \mathrm{SO}_{4}(70 \mathrm{~mL})$. Then, $\mathrm{NaNO}_{3}(1.5 \mathrm{~g})$ was added to the mixture. The mixture was cooled to $0^{\circ} \mathrm{C} . \mathrm{KMnO}_{4}(9.0 \mathrm{~g})$ was added slowly under vigorous agitation to ensure that the temperature of the suspension was lower than $20^{\circ} \mathrm{C}$. Subsequently, the mixture was stirred at $35^{\circ} \mathrm{C}$ for $2 \mathrm{~h}$. Distilled water $(150 \mathrm{~mL})$ was added to the mixture, and the solution was stirred at $90^{\circ} \mathrm{C}$ for $15 \mathrm{~min}$.

An additional $500 \mathrm{~mL}$ of distilled water was added to the mixture, and then $15 \mathrm{~mL}$ of $\mathrm{H}_{2} \mathrm{O}_{2}$ (3\% volume) was added. Filtration and washing with a $1: 10 \mathrm{HCl}$ aqueous solution $(250 \mathrm{~mL})$ were performed to remove the metal ions, and distilled water $(200 \mathrm{~mL})$ washing was carried out to remove the acid. The obtained solid was dried in air and then diluted to produce $\mathrm{GO}$ aqueous dispersion ( $0.5 \mathrm{wt} . \%)$. Sonication was used for the dissolution of $1 \mathrm{~g}$ of the dried GO in $20 \mathrm{~mL}$ of deionized water to obtain yellow homogeneous dispersion.

2.2.2. Reduction of GO by Chemical Reduction. In total, $40 \mu \mathrm{L}$ of hydrazine hydrate $(\mathrm{HH})$ (reducing agent) was added to the GO solution. This solution was placed inside a conventional microwave. We used the microwave at full power $(1000 \mathrm{~W})$, $2.45 \mathrm{GHz}$, with $30 \mathrm{~s}$ cycles (for $10 \mathrm{~s}$, off, and stirring for $20 \mathrm{~s}$ ) for 60 seconds in total, which was the total reaction time. The yellow dispersion of GO changed to a black color, which indicates that the chemical reduction to graphene occurred.
A centrifuge was used to separate the graphene sheets, which was operated at $5000 \mathrm{rpm}$ for $15 \mathrm{~min}$. Following centrifugation, the sample was dried overnight under vacuum.

2.2.3. Bacterial Cultures and Graphene Reduction Using the Biotechnological Method. All glassware and samples used in the biotechnological method were sterilized by autoclaving at $120^{\circ} \mathrm{C}$ for $15 \mathrm{~min}$. Bacteria were cultured on a nutrient agar plate for $24 \mathrm{~h}$ at $37^{\circ} \mathrm{C}$. E. coli bacteria were inoculated from frozen stocks into nutrient broth (NB), and the bacterial cultures were grown overnight at room temperature. To remove all traces of $\mathrm{NB}$, the overnight cultures were washed with saline solution. Subsequently, the cultured bacteria were transferred to a serum bottle containing $10 \mathrm{~mL}$ of nutrient broth $(\mathrm{NB})$ to reach a bacterial concentration of approximately $10^{4}$ colony forming units (CFU)/mL. The $\mathrm{pH}$ of the medium was adjusted to 7 by using $\mathrm{NaOH}$. Then, GO was used to prepare the medium. The film that contained a bacterial suspension was incubated at $37^{\circ} \mathrm{C}$ in the dark. Control samples that contained only GO and media were used as a reference. All of the samples were kept at room temperature. After 20 days, the samples were washed with saline and characterized.

This technique is different than that presented in the published work by Gurunathan et al. [8], as this group produced graphene suspended in water and used a high temperature, while, herein, we report the production of graphene at room temperature.

2.3. Characterization. XRD, Raman spectroscopy, SEM, and XPS were used to characterize the chemically and biotechnologically reduced graphene samples and to obtain information about chemical and surface changes.

2.3.1. X-Ray Diffraction (XRD). XRD patterns of the prepared graphite, graphene oxide, and graphene (both samples prepared using chemical and bacterial reduction methods) were analyzed using a powder X-ray diffractometer (Rigaku Miniflex 2 Desktop). The XRD patterns were recorded using $\mathrm{Cu}$ radiation at $30 \mathrm{kV}, 15 \mathrm{~mA}$, in the range of $2 \theta=5^{\circ}-70^{\circ}$. The continuous scan mode was used, and $2^{\circ} / \mathrm{min}$ was used as the scan speed.

2.3.2. Raman Spectroscopy. Raman spectroscopy was performed at room temperature using a confocal Raman microspectrometer (inVia Reflex, Renishaw) with different excitation lines $(532 \mathrm{~nm}, 633 \mathrm{~nm}$, and $785 \mathrm{~nm})$. Without any pretreatment, the samples were measured directly and the laser power was $\sim 2 \mathrm{~mW}$. The presented spectra were obtained as an average spectrum of 5 different parameters of each registration using a spectral range of $100-3500 \mathrm{~cm}^{-1}$ with 3 accumulations and an exposure time of $10 \mathrm{~s}$.

2.3.3. X-Ray Photoelectron Spectroscopy (XPS) Analysis. XPS analysis was performed using a Thermo Scientific K-Alpha 


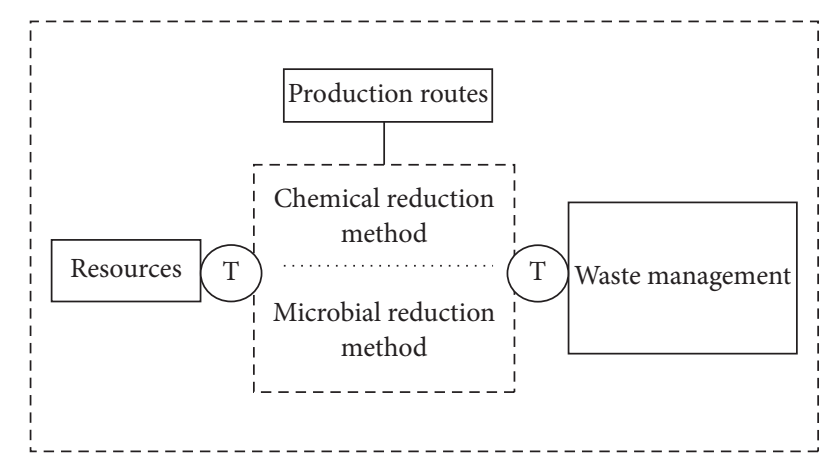

FIGURE 1: The scheme of the LCA scenario for graphene production.

XPS system with a micro focused monochromatic $\mathrm{Al} \mathrm{K} \alpha \mathrm{X}$ ray source $(1486.6 \mathrm{eV})$. The constant analyzer energy mode and a pass energy of $200 \mathrm{eV}$ survey were employed. The beam size was $400 \mu \mathrm{m}$ at $6 \mathrm{~mA} \times 12 \mathrm{kV}$. The snapshot acquisition mode was used to collect narrow regions with a $150 \mathrm{eV}$ pass energy, enabling the rapid collection of data ( $5 \mathrm{~s}$ per region). Charge compensation was achieved with the system flood gun that provides low energy electrons $(\sim 0 \mathrm{eV})$ and low energy argon ions $(20 \mathrm{eV})$ from a single source. The argon partial pressure was $2 \times 10^{-7} \mathrm{mbar}$ in the analysis chamber.

\subsubsection{Morphological Analysis (Scanning Electron Microscopy} $(S E M))$. The morphological analyses of GO and graphene were carried out via scanning electron microscopy (Nova NanoSEM 450, FEI, USA), using a secondary electron detector. All samples were analyzed at several locations to identify characteristic and significant surface features.

2.3.5. Life Cycle Assessment (LCA). Life cycle assessment was studied by $\mathrm{GaBi}$ software. This study was focused on the two methods of preparing graphene by the reduction of $\mathrm{GO}$, which was produced from graphite (raw material). The functional unit is the procedure required to prepare $1 \mathrm{~kg}$ of graphene. The system boundaries are established according to the commonly used "cradle to gate" system; these boundaries involve the chemicals that are used for the perpetration. The process starts with graphite production and involves stirring, filtration, drying, sonication, and centrifugation of the samples obtained by the chemical reaction to produce graphene. The second process, biotechnological reduction, includes fewer preparation steps that can reduce the energy consumption, but this process consumes more water. The general scheme of the LCA system boundaries is shown in Figure 1.

The range of the system boundaries in this study uses Hummers' process as a baseline.

In Hummers' process, the graphite oxide is reduced by hydrazine, which is a chemical reduction. The biotechnological reduction employed caused the bacterial suspension to reduce the graphite oxide. Both methods will be compared, as the preparation steps are different from each other after the stirring step.

\section{Results and Discussion}

3.1. XRD Analysis. The XRD patterns of graphite, graphene oxide, chemically reduced graphene, and graphene produced by $E$. coli are shown in Figure 2. The XRD pattern of graphite in Figure 2(a) shows a strong sharp diffraction peak at $2 \theta=$ $26.56^{\circ}$ that corresponds to interlayer spacing of $3.35 \AA$. The XRD spectrum of graphene oxide in Figure 2(b) has a peak at $10.82^{\circ}$ and corresponds to interlayer spacing of $8.17 \mathrm{~A}^{\circ}$, which confirms the oxidation of graphene, and is significantly larger than that of graphite. This result is similar to reported values in the literature [11]. The increase in spacing is due to the formation of oxygen contacting functional groups between the graphite layers. Another peak appeared at $25.92^{\circ}$ with interlayer spacing of $3.434 \AA$.

The peak at $10.82^{\circ}$ is not shown in the XRD pattern of chemically produced graphene (Figure $2(\mathrm{c})$ ). The disappearance of this peak confirms the reduction of GO to graphene [11]. The presence of the broad peak at $24.96^{\circ}$ in graphene indicates the presence of only a few layers of graphene [12]. This peak corresponds to the 002 plane of graphite with interlayer spacing of $3.56 \AA$ that is due to the removal of oxygen atoms that entered the graphite gallery during the intercalation process. This confirms the reduction of GO to graphene using a chemical reduction method. The reduction of $\mathrm{GO}$ with bacteria causes the disappearance of the peak at $10.82^{\circ}$ and a broad peak appears at approximately $24 \AA$, as shown in Figure 2(d). The interlayer spacing decreases, which suggests that exfoliation caused oxygen and water to be removed from the interlayer to a large extent [12].

3.2. Raman Spectroscopy. Raman spectroscopy is an important and simple method used to characterize the number of graphene layers produced. Graphene is generally characterized using Raman spectroscopy by studying two main features: the G-peak (approximately $1575 \mathrm{~cm}^{-1}$ ) and D-peak (approximately $1350 \mathrm{~cm}^{-1}$ ) that arise due to the graphitized structure and the local defects/disorders found at the edges of the graphene [13], respectively. The Raman spectra of graphite and graphene oxide are shown in Figures 3(a) and 3(b), respectively. Notable structural changes occur during the processing of GO. The Raman spectrum of the graphite, as expected, displays a prominent G-peak as its only feature at $1577 \mathrm{~cm}^{-1}$, corresponding to the first-order scattering of the E2g mode [9]. The G-band broadens and shifts to $1604 \mathrm{~cm}^{-1}$ in the Raman spectrum of GO [14].

In addition, the $\mathrm{D}$-band at $1364 \mathrm{~cm}^{-1}$ becomes prominent and indicates a reduction in the size of the in-plane $\mathrm{sp}^{2}$ domains, which is possible following extensive oxidation. Figures 3(c) and 3(d) show the Raman spectra of graphene, which were reduced by chemical reduction and bacterial reduction methods, respectively. The Raman spectrum of graphene has both G- and D-bands (approximately at 1584 and $1356 \mathrm{~cm}^{-1}$, resp.); a new band at $2700 \mathrm{~cm}^{-1}$ is shown in Figures $3(\mathrm{c})$ and $3(\mathrm{~d})$. This band is called the $2 \mathrm{D}$ band, and it is observed only in graphene structures.

The ratio of $I_{2 D} / I_{G}$ of the intensity depends on the number of graphene layers. The ratio $I_{2 D} / I_{G}<1$ in both types of 


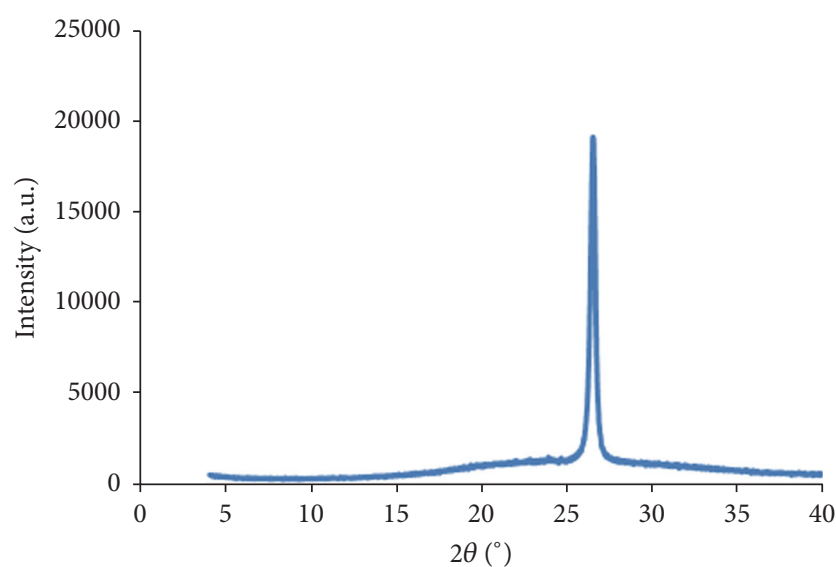

(a)

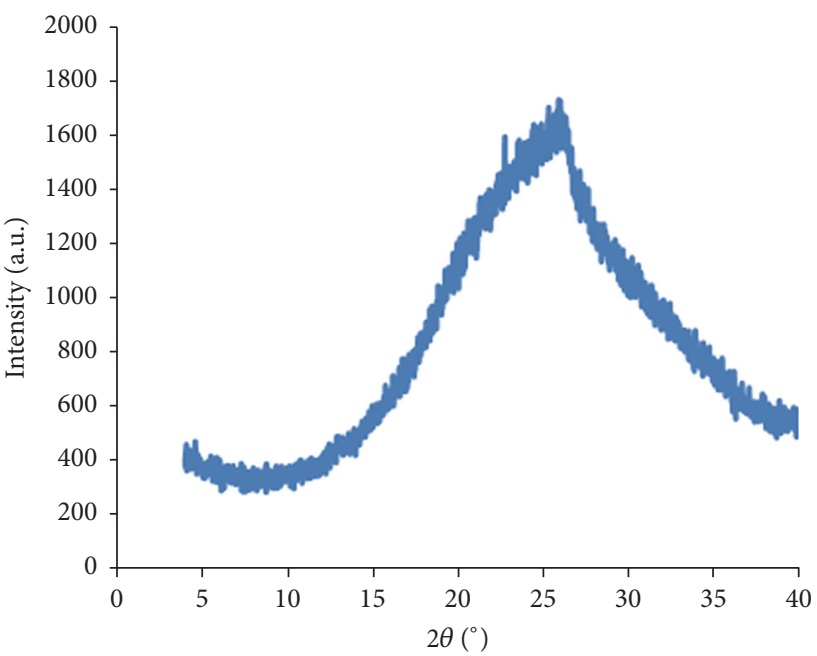

(c)

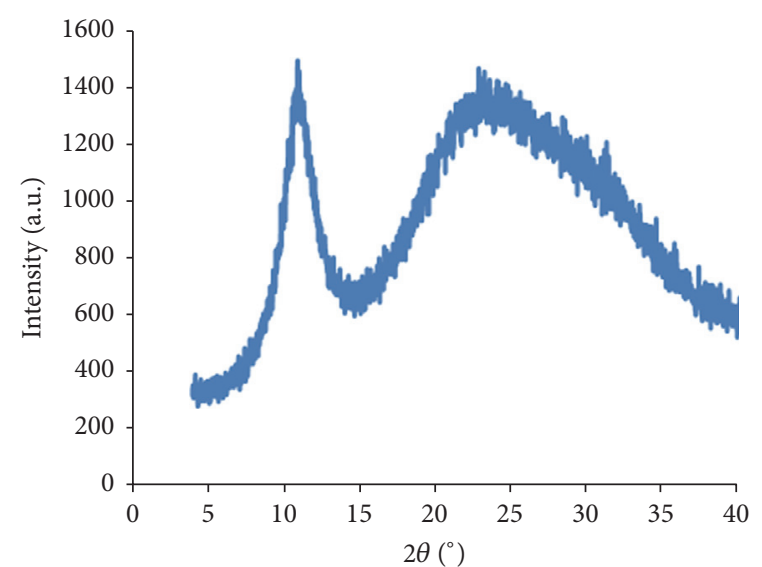

(b)

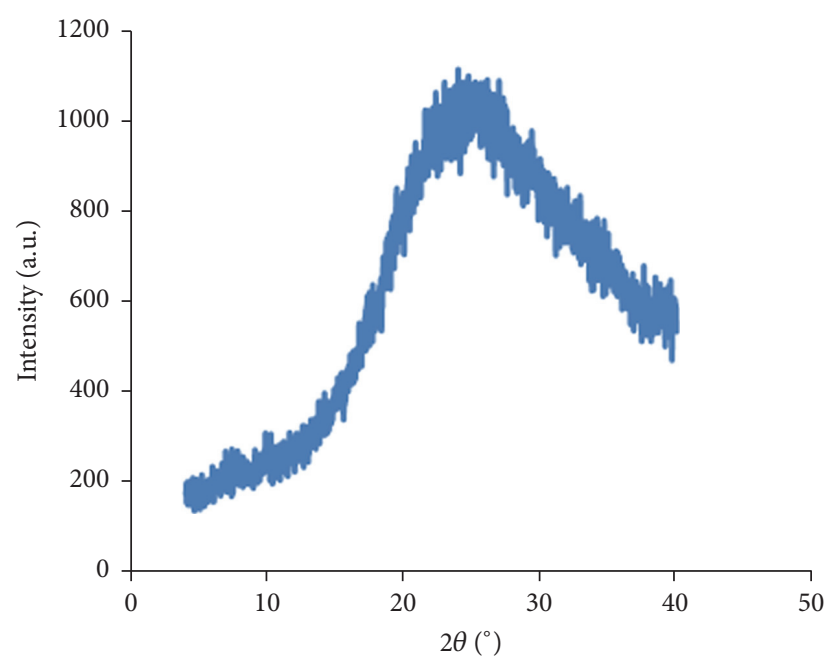

(d)

FIGURE 2: (a) XRD pattern of graphite. (b) XRD pattern of graphene oxide. (c) XRD pattern of graphene which is prepared by chemical reduction method. (d) XRD of graphene which is prepared by using E. coli bacteria.

graphene, which indicates multilayer graphene production. The slight increase in this intensity for the biotechnologically produced graphene indicates a smaller number of graphene layers.

3.3. XPS Analysis. XPS was used to confirm the chemical composition of the prepared samples. The XPS survey-scan spectra of all samples are shown in Figure 4. The chemical compositions of GO and graphene samples that were prepared by the chemical reduction and the biotechnological reduction method are shown in Figure 4. The spectrum of GO (Figure 4(a)) was characterized predominantly by peaks centered at binding energies of $285 \mathrm{eV}$ and $532 \mathrm{eV}$ corresponding to $\mathrm{Cls}$ and $\mathrm{O} 1 \mathrm{~s}$, respectively. The high resolution of the Cls peak was attributed to $\mathrm{C}-\mathrm{C}\left(\mathrm{sp}^{2}, \mathrm{sp}^{3}\right.$, and $\pi$ $\left.\pi^{*}\right)$ as well as $\mathrm{C}-\mathrm{O}, \mathrm{C}=\mathrm{O}$, and $\mathrm{O}-\mathrm{C}=\mathrm{O}$ (Figure $5(\mathrm{a})$ ). The $\mathrm{GO}$ contained the highest proportional content of oxygen compared to other samples. Moreover, small traces of the N1s, S2p, Cl2p, and Si2p peaks were presented in the GO spectrum as a result of some impurities introduced during sample preparation, which likely originated from the reaction environment or the chemicals used. The XPS was also used to confirm the reduction of GO, as shown in Figures 4(b) and $4(\mathrm{c})$ and in the high-resolution Cls spectra (Figures 5(b) and 5(c)), which were prepared by both the chemical and the biotechnological reduction methods. The intensity of the Ols peak decreased remarkably, indicating the successful removal of oxygen groups for both of the employed reduction methods. The largest reduction of GO was observed in the use of the chemical method, but the biotechnological method also confirmed a notable reduction effect. The production of graphene is likely accomplished due to the electron mediators involved [8], but further studies are needed to elucidate the exact details and mechanisms of the production of graphene.

3.4. Scanning Electron Microscopy. Figures 6(a) and 6(b) show the SEM images of graphite and GO, respectively. SEM images of graphite show flaky shape and irregular, thicker platelets, which are present in the powder. SEM images of GO (Figure 6(b)) show hexagonal shape and layered GO 


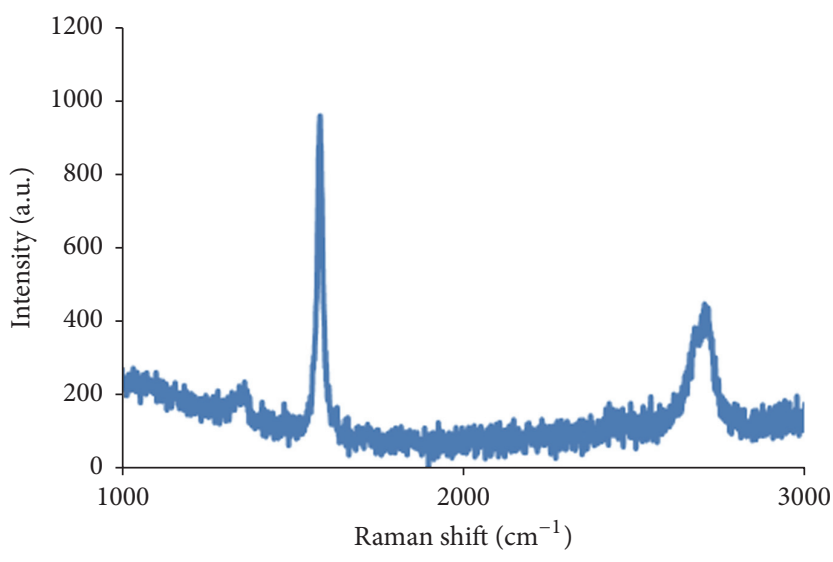

(a)

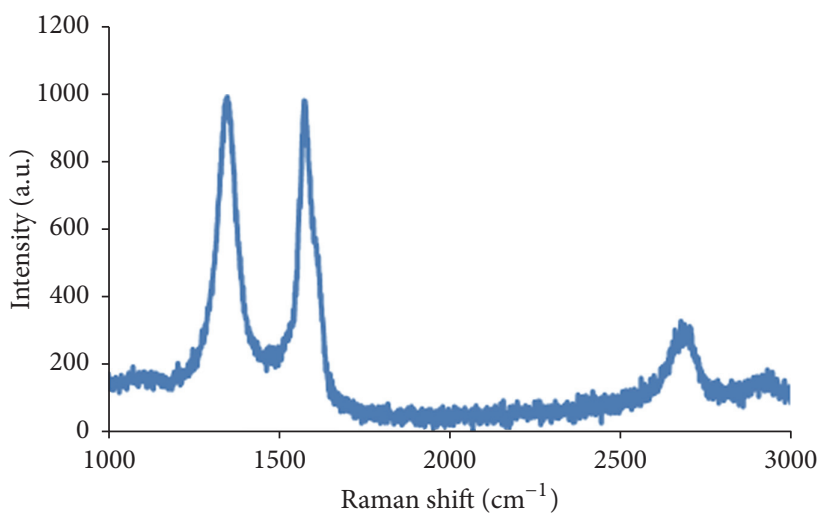

(c)

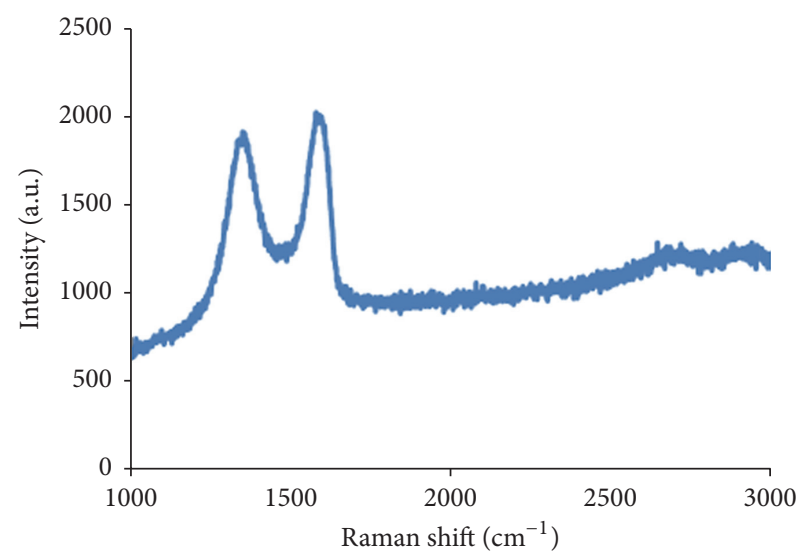

(b)

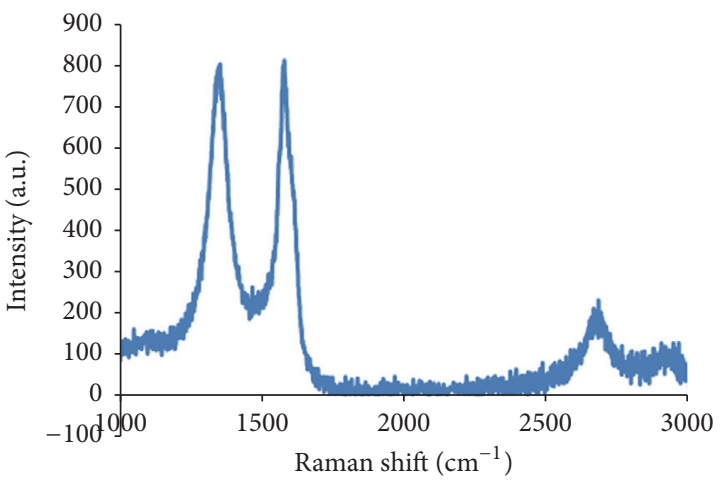

(d)

Figure 3: (a) Raman spectra of graphite. (b) Raman spectra of graphene oxide. (c) Raman spectra of graphene prepared by using chemical reduction method. (d) Raman spectra of graphene prepared by E. coli.

flakes, which are smaller than graphite. Pi stacking led to the agglomeration of these flakes.

SEM images of chemically reduced graphene (Figure 6(c)) and biotechnologically reduced graphene (Figure $6(\mathrm{~d})$ ) revealed that the graphene consists of thin randomly aggregated and crumpled sheets that are closely associated with each other and form a disordered solid. The appearances of the graphene sheet petals are observed to agglomerate, most likely because of their small size [15]. The surface of the graphene is not perfectly flat but contains wrinkles that give different levels of transparency. The layers cannot be clearly observed by SEM. However, the layers can be distinguished through the folded edge and graphene flakes are overlapped in the SEM image of graphene, which is produced by the chemical reduction method [16]. The SEM of biotechnologically reduced graphene also shows folded flakes and a smaller amount of aggregated material (Figure 6(d)).

3.5. Life Cycle Impact Assessment (LCIA). A life cycle impact assessment is the method used to explain the environmental impact of the produced graphene. Many factors, based on the inherent characteristics of chemicals involved in the sample preparation and information about transportation and exposure, can affect the production of graphene [17]. The life cycle impact assessment methodology based on the CML 2001 issue from Nov. 10 and developed by the Institute of Environmental Sciences in Leiden (Netherlands) is used in this study. The investigation of environmental impacts was broadened to include the selected impact categories from Table 1. The evaluation of the environmental impacts for graphene produced by both (1) the chemical method and (2) the biotechnological method is shown in Figure 7.

3.5.1. Life Cycle Interpretation and Discussion. The objective of this study was to demonstrate the life cycle impacts of all processes and flows in order to establish the relative environment impacts of graphene production. Different impact categories were investigated for both methods, as shown in Figure 7. The main difference between the two methods is the consumption of energy during each production process. The same process of waste management was applied for both methods.

Graphene production by chemical reduction is considered as a high energy consumption process due to the required heat and also because of the reaction of hydrazine hydrate $(\mathrm{HH})$. In the case of the biotechnological reduction process, the energy consumption is less because the conversion process occurs at room temperature without supplying 


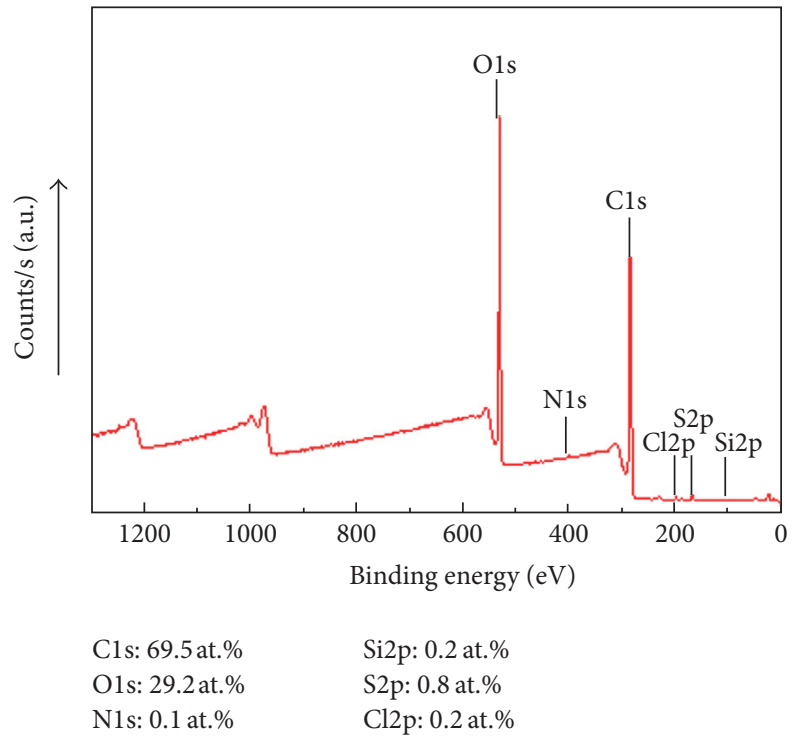

(a)

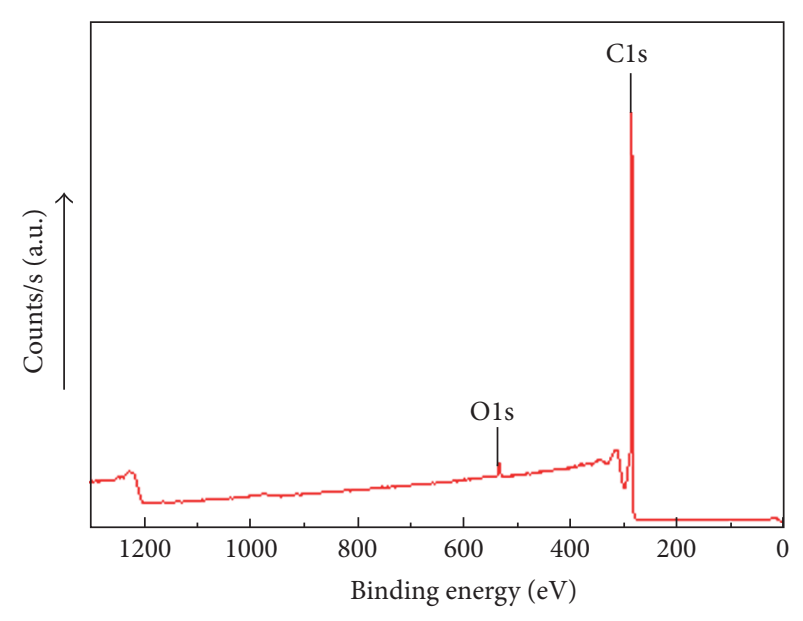

C1s: 98.2 at.\%

O1s: 1.8 at.\%

(b)

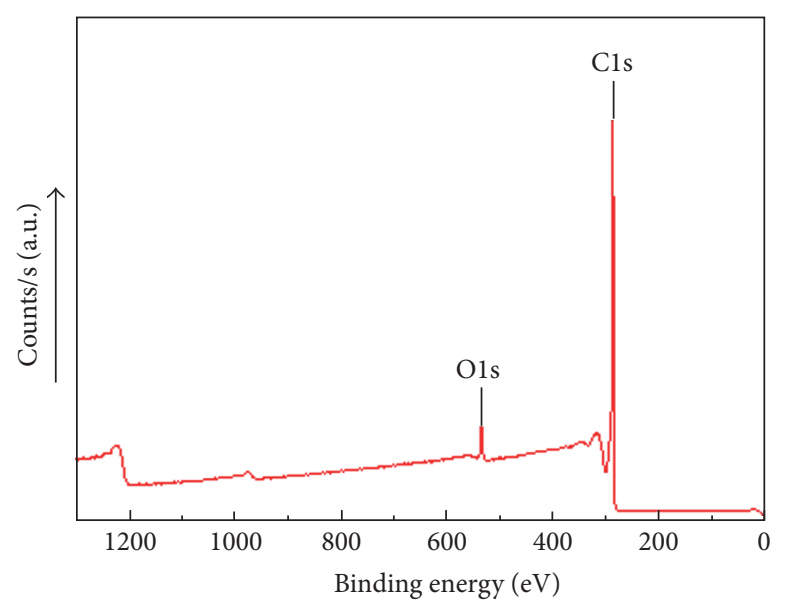

C1s: 95.5 at.\%

O1s: 4.5 at.\%

(c)

FIGURE 4: (a) XPS spectra of graphene oxide. (b) XPS spectra of graphene prepared by chemical reduction method. (c) XPS spectra of graphene prepared by using $E$. coli bacteria.

TABLE 1: List of impact categories used in this study.

\begin{tabular}{lcc}
\hline Impact category & Characterization factor & Indicator unit \\
\hline Climate change & Global warming potential (GWP 100 year) & $\mathrm{kg}\left(\mathrm{CO}_{2}\right.$ eq. $)$ \\
Acidification & Acidification potential (AP) & $\mathrm{Kg}\left(\mathrm{SO}_{2}\right.$ eq. $)$ \\
Eutrophication & Eutrophication potential (EP) & $\mathrm{kg}\left(\mathrm{PO}_{4}\right.$ eq.) \\
Stratospheric ozone depletion & Ozone depletion potential (ODP) & $\mathrm{kg}(\mathrm{R} 11 \mathrm{eq})$. \\
Abiotic depletion & Abiotic potential elements (APE) & $\mathrm{kg}(\mathrm{Sb}$ eq.) \\
Abiotic depletion & Abiotic potential fossil (APF) & $\mathrm{MJ}$ \\
Freshwater aquatic ecotoxicity & Freshwater aquatic ecotoxicity potential (FAETP) & $\mathrm{kg}(\mathrm{DCB}$ eq.) \\
Human toxicity & Human toxicity potentials (HTP) & $\mathrm{kg}(\mathrm{DCB}$ eq.) \\
Marine aquatic ecotoxicity & Marine aquatic ecotoxicity potential (MAETP) & $\mathrm{kg}(\mathrm{DCB}$ eq.) \\
Terrestrial ecotoxicity & Terrestrial ecotoxicity potential (TETP) & $\mathrm{kg}(\mathrm{DCB}$ eq.) \\
Photooxidant formation & Photochemical ozone creation potential (POCP) & $\mathrm{kg}\left(\mathrm{C}_{2} \mathrm{H}_{4}\right.$ eq.) \\
\hline
\end{tabular}




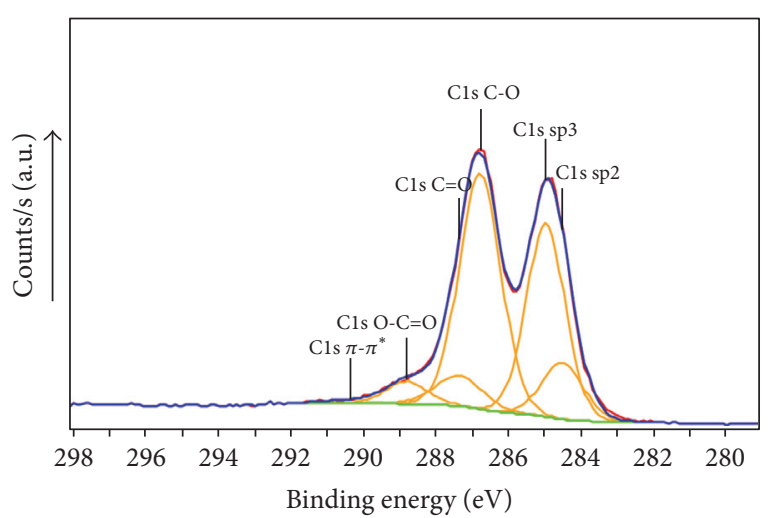

(a)

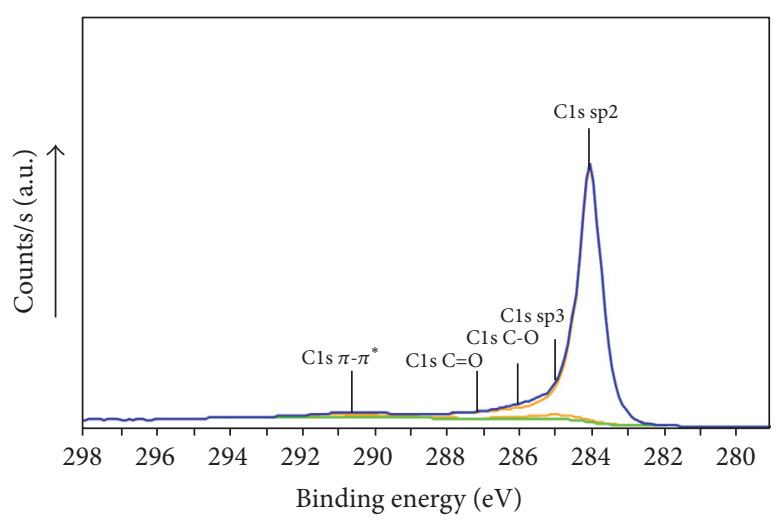

(b)

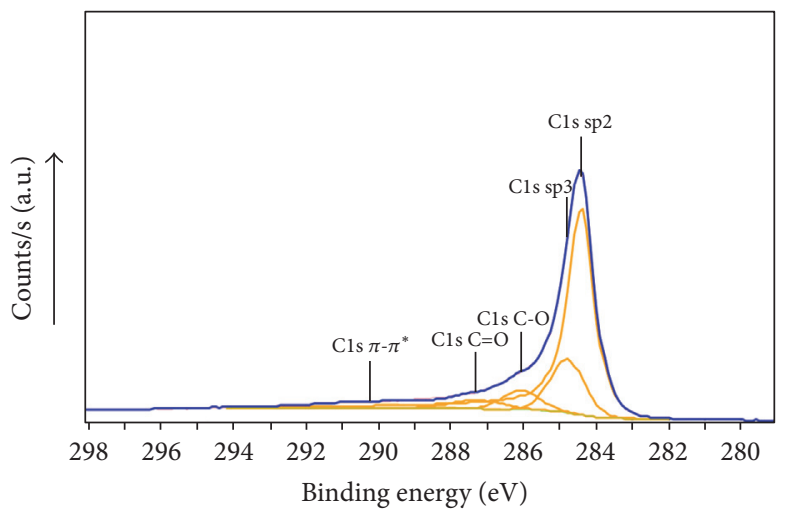

(c)

FIGURE 5: (a) High-resolution C1s spectra of graphene oxide. (b) High-resolution C1s spectra of graphene prepared by chemical reduction method. (c) High-resolution C1 spectra of graphene prepared by E. coli bacteria.

any additional energy. Arvidsson et al. [9] compared the LCA of the chemical reduction route and ultrasonication route for the graphene production, and they reported that high energy is required for the production of graphene prepared using the chemical reduction method. They reported that the energy used in the chemical reduction method is approximately twice that of the ultrasonication route because of heating processes that are required in the chemical reduction process.

We did not consider the ultrasonication procedure for use in preparing graphene in this study, as Arvidsson et al. [9] explained that the ultrasonication route has the largest impact (about three times the chemical method) on humans due to its toxicity.

In the present study, the energy used for the chemical reduction process is approximately $1642 \mathrm{Wh}$, which is higher than that in the biotechnological reduction process $(5 \mathrm{Wh})$, as there is less electricity consumption.

Compared to other carbon materials such as CNT, the production of graphene by the chemical reduction method requires less energy [9].

The impact of climate change is represented by the potential of global warming (GWP 100) for a time horizon of 100 years, which can be related to the resulting greenhouse emission from the electricity production. The electricity production and transportation can be considered a background system that is important in environmental evaluation but was excluded in some studies [9]. The foreground is the system of the investigated product. The current study includes both the background and the foreground. GWP 100 is changed by production via the biological method by $71 \%$ compared to the chemical method of production.

Another important environmental parameter is the acidification, which is dominated by the release of sulfur dioxide $\left(\mathrm{SO}_{2}\right)$, which has the same value produced through the production of graphene oxide. The $\mathrm{HH}$ production of the chemical reduction is the main reason for the increase in the acidification potential in this case as it causes the production of $\mathrm{NO}_{x}$ species. The reduction process by the microbial method has a lessened impact compared with the chemical reduction, as the production of $\mathrm{NO}_{x}$ does not occur.

Both the chemical and the biological methods have the same effect on the eutrophication potential, which is measured mainly by the amount of phosphorus as well as the amount of $\mathrm{NO}_{x}$ and $\mathrm{NH}_{3}$. This is due to the GO production effect, which is the same for both graphene production procedures.

The ozone depletion potential provides information about the ozone loss expected for the selected material. It causes a thinner stratospheric ozone layer. Both reduction methods have approximately the same value for the ozone 


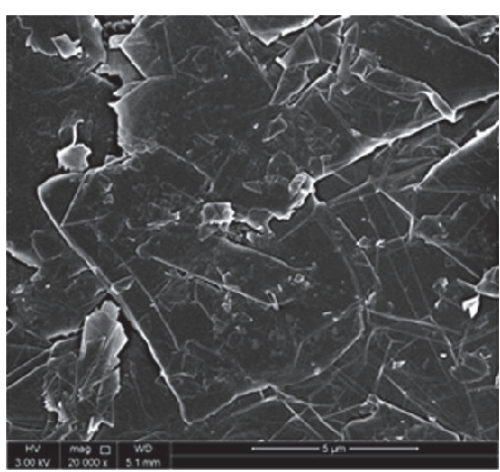

(a)

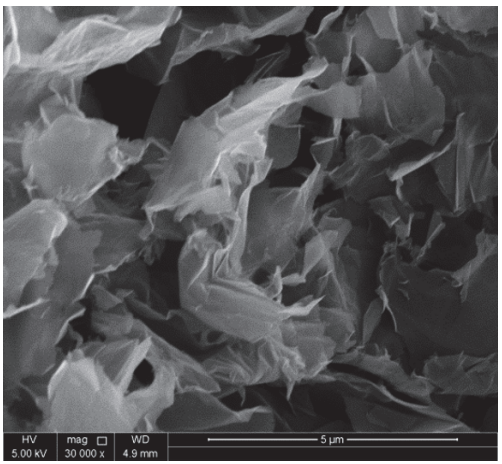

(d)

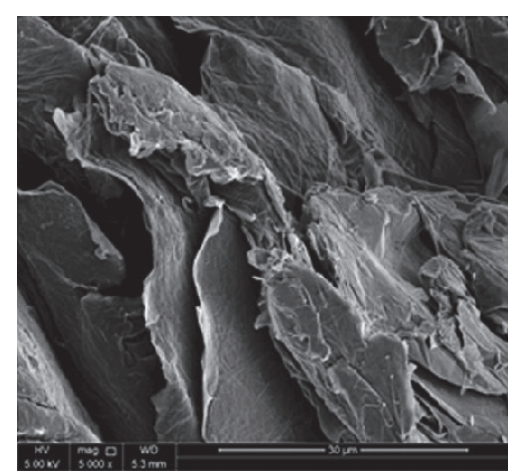

(b)

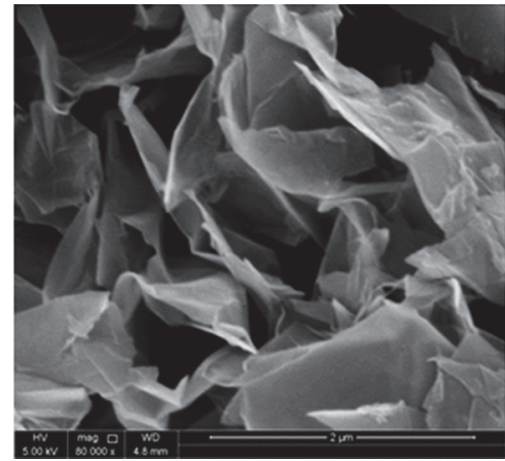

(e)

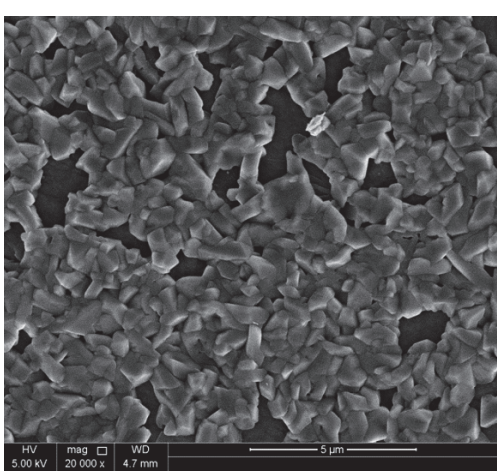

(c)

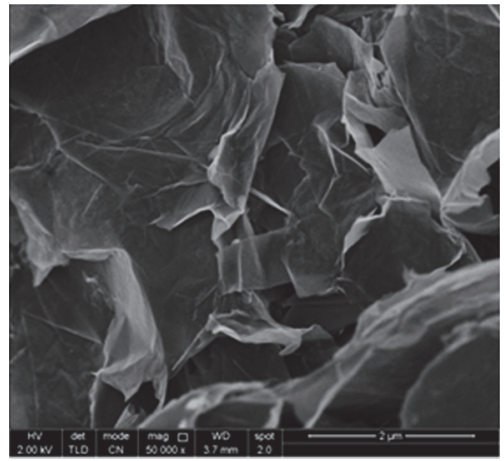

(f)

FIGURE 6: SEM images of (a) pure graphite, (b) pure graphene oxide, and (c) graphene prepared by chemical reduction method and (d) graphene prepared by microbial reduction method. (e) and (f) are higher magnifications of (c) and (d).

depletion layer as the variables affecting the production of GO responsible for the depletion as well as for the waste management are the same.

The abiotic depletion consists of the reduction of mineral and energy resources [17]. Biotechnological reduction has lessened abiotic depletion due to the decrease in consumption of fuel and electricity.

Both the chemical and the biotechnological methods have the same effect on the freshwater aquatic toxicity potential and the human toxicity potential, as the used variables are from similar sources ( $\mathrm{GO}$ production and waste management process).

A slight increase in the marine aquatic ecotoxicity potential (MAETP) (7.6\%) is due to the extra use of electricity in the chemical method. The main source for MATEP is the waste management process, which is the same for both production methods.

The photochemical ozone creation potential is measured by the volatile organic compounds that are emitted in the atmosphere. Slight improvement is noticed for the biotechnological production route due to the high energy consumption and use of the $\mathrm{HH}$ chemical in the chemical route of graphene production.

Terrestrial ecotoxicity, which is related to metals and metalloids, is approximately the same for the biotechnological and the chemical reduction of graphene due to the same source, which is, namely, the management process.

\section{Conclusions}

Heavily oxygenated graphene oxide was simply reduced to graphene by the following two methods: (1) a common chemical method and (2) a biotechnological method. XRD and Raman spectroscopy showed that the graphene produced by both methods was similar, with more layers produced by the chemical method compared to the biotechnological method. XPS confirmed the significant reduction of oxygen content from 29.2 at.\% in graphene oxide to 4.5 at.\% and 1.8 at.\% in the method utilizing E. coli and the chemical method, respectively.

The formation of graphene by the biotechnological method proved to be more environmentally friendly, especially with respect to the following parameters: the potential of global warming (GWP 100), abiotic depletion, the photochemical ozone creation potential, and the marine aquatic ecotoxicity potential.

\section{Disclosure}

The statements made herein are solely the responsibility of the authors. 


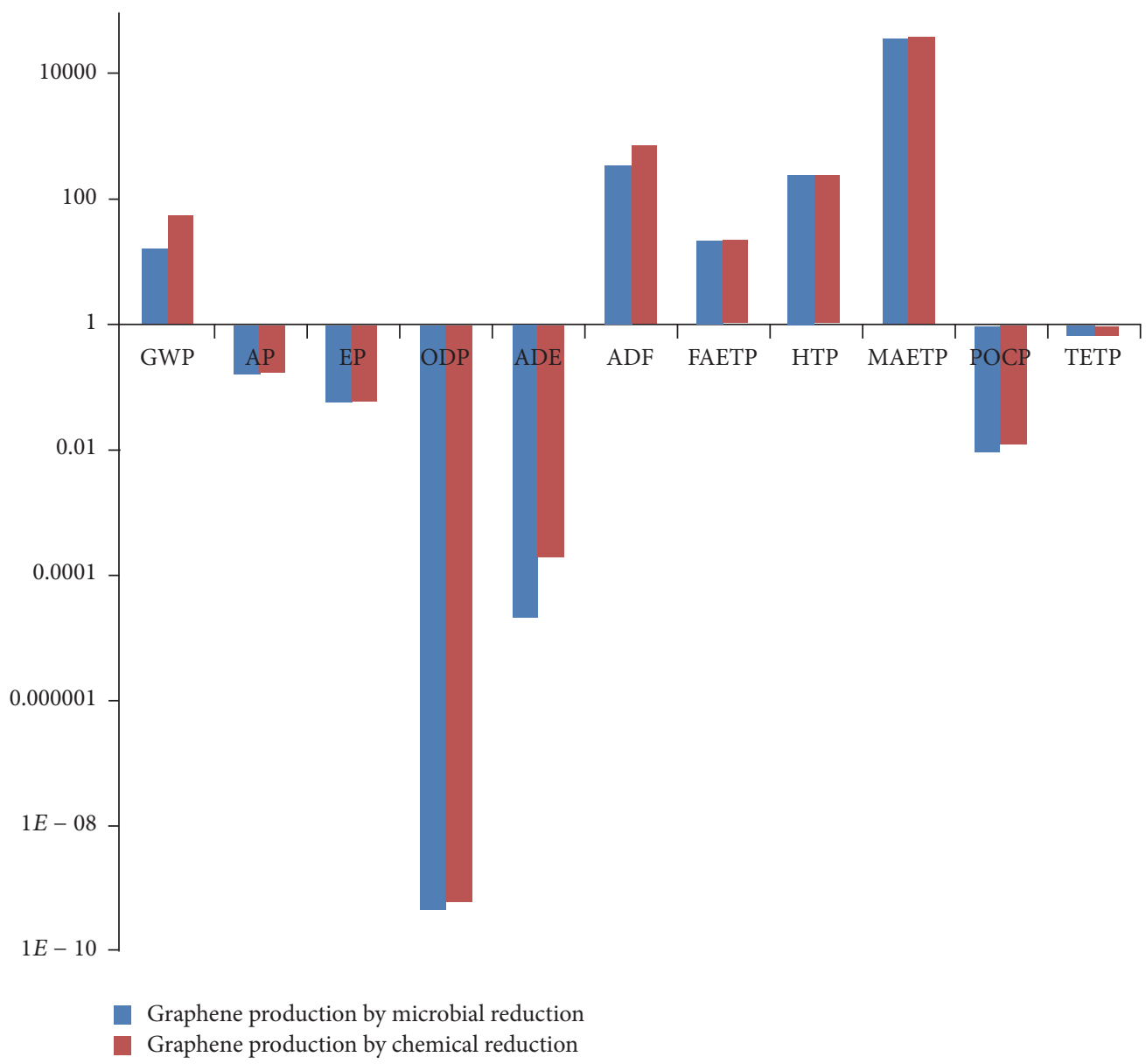

FIGURE 7: The environmental impact assessment for both methods of graphene production.

\section{Competing Interests}

The authors declare that there are no competing interests regarding the publication of this paper.

\section{Acknowledgments}

This article was made possible by NPRP Grant no. NPRP5039-2-014 from the Qatar National Research Fund (a member of Qatar Foundation). The authors would like to thank Dr. Roda AlThani from Qatar University for the fruitful discussion.

\section{References}

[1] D. Li, M. B. Müller, S. Gilje, R. B. Kaner, and G. G. Wallace, "Processable aqueous dispersions of graphene nanosheets," Nature Nanotechnology, vol. 3, no. 2, pp. 101-105, 2008.

[2] T. Kuilla, S. Bhadra, D. Yao, N. H. Kim, S. Bose, and J. H. Lee, "Recent advances in graphene based polymer composites," Progress in Polymer Science, vol. 35, no. 11, pp. 1350-1375, 2010.

[3] M. Zhou, Y. Zhai, and S. Dong, "Electrochemical sensing and biosensing platform based on chemically reduced graphene oxide," Analytical Chemistry, vol. 81, no. 14, pp. 5603-5613, 2009.
[4] S. Myung, A. Solanki, C. Kim, J. Park, K. S. Kim, and K.-B. Lee, "Graphene-encapsulated nanoparticle-based biosensor for the selective detection of cancer biomarkers," Advanced Materials, vol. 23, no. 19, pp. 2221-2225, 2011.

[5] C. Gómez-Navarro, R. T. Weitz, A. M. Bittner et al., "Electronic transport properties of individual chemically reduced graphene oxide sheets," Nano Letters, vol. 7, no. 11, pp. 3499-3503, 2007.

[6] O. Akhavan and E. Ghaderi, "Toxicity of graphene and graphene oxide nanowalls against bacteria," ACS Nano, vol. 4, no. 10, pp. 5731-5736, 2010.

[7] Y. Tanizawa, Y. Okamoto, K. Tsuzuki et al., "Microorganism mediated synthesis of reduced graphene oxide films," Journal of Physics: Conference Series, vol. 352, no. 1, 2012.

[8] S. Gurunathan, J. W. Han, V. Eppakayala, and J.-H. Kim, "Microbial reduction of graphene oxide by Escherichia coli: a green chemistry approach," Colloids and Surfaces B: Biointerfaces, vol. 102, pp. 772-777, 2013.

[9] R. Arvidsson, D. Kushnir, B. A. Sandén, and S. Molander, "Prospective life cycle assessment of graphene production by ultrasonication and chemical reduction," Environmental Science \& Technology, vol. 48, no. 8, pp. 4529-4536, 2014.

[10] V. K. K. Upadhyayula, D. E. Meyer, M. A. Curran, and M. A. Gonzalez, "Life cycle assessment as a tool to enhance the environmental performance of carbon nanotube products: a review," Journal of Cleaner Production, vol. 26, pp. 37-47, 2012. 
[11] C. Teng, C. M. Ma, C. Lu et al., "Thermal conductivity and structure of non-covalent functionalized graphene/epoxy composites," Carbon, vol. 49, no. 15, pp. 5107-5116, 2011.

[12] M. Wojtoniszak, X. Chen, R. J. Kalenczuk et al., "Synthesis, dispersion, and cytocompatibility of graphene oxide and reduced graphene oxide," Colloids and Surfaces B: Biointerfaces, vol. 89, pp. 79-85, 2012.

[13] O. Akhavan and E. Ghaderi, "Escherichia coli bacteria reduce graphene oxide to bactericidal graphene in a self-limiting manner," Carbon, vol. 50, no. 5, pp. 1853-1860, 2012.

[14] K. N. Kudin, B. Ozbas, H. C. Schniepp, R. K. Prud'homme, I. A. Aksay, and R. Car, "Raman spectra of graphite oxide and functionalized graphene sheets," Nano Letters, vol. 8, no. 1, pp. 36-41, 2008.

[15] Y. Chen, H. Zhao, L. Sheng et al., "Mass-production of highlycrystalline few-layer graphene sheets by arc discharge in various $\mathrm{H}_{2}$-inert gas mixtures," Chemical Physics Letters, vol. 538, no. 11, pp. 72-76, 2012.

[16] G. Sobon, J. Sotor, J. Jagiello et al., "Graphene Oxide vs. Reduced Graphene Oxide as saturable absorbers for Er-doped passively mode-locked fiber laser," Optics Express, vol. 20, no. 17, pp. 19463-19473, 2012.

[17] J. B. Guinee, Handbook on Life Cycle Assessment, Operational Guide to the ISO Standards, Kluwer Academic, Dordrecht, The Netherlands, 2002. 

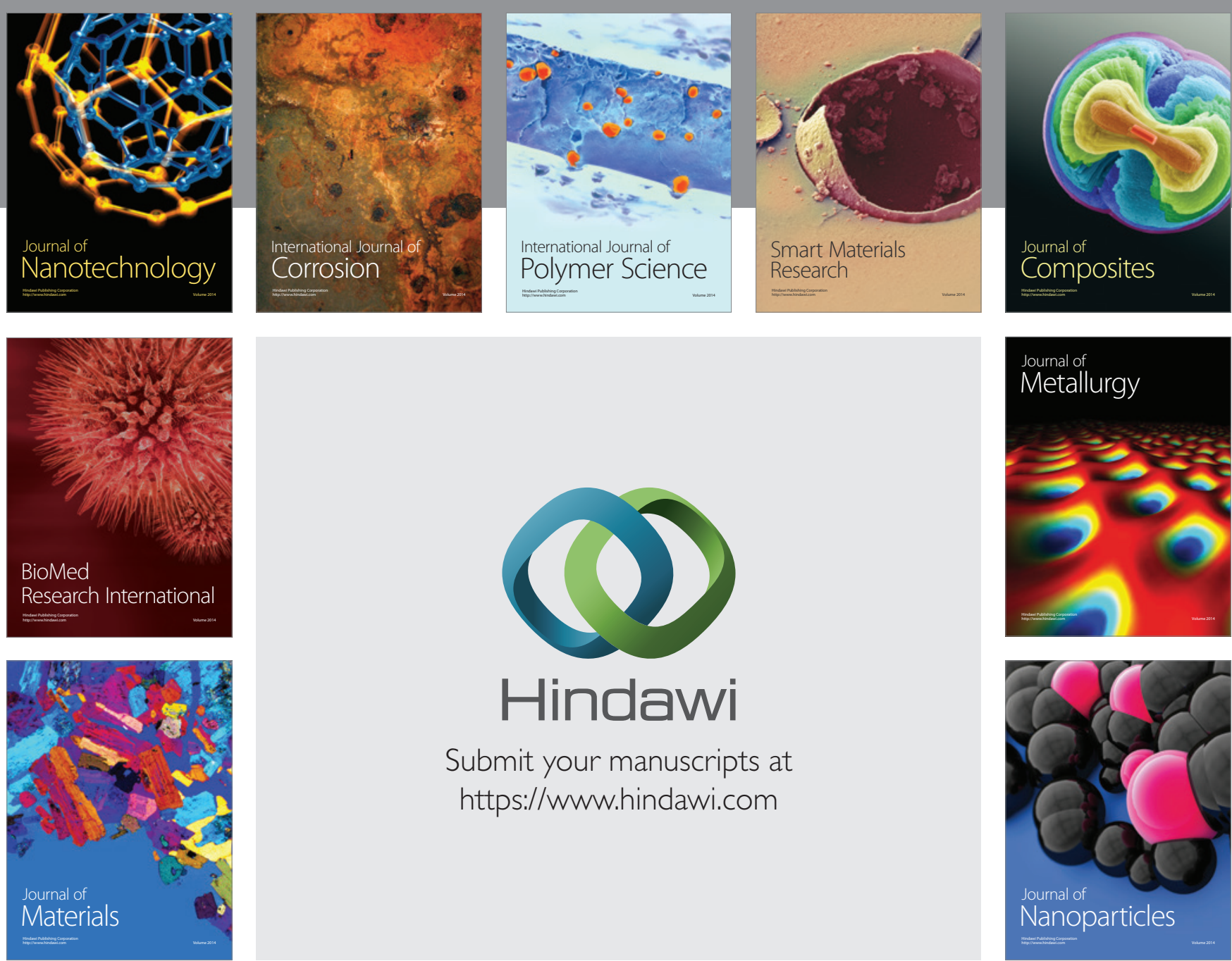

\section{Hindawi}

Submit your manuscripts at

https://www.hindawi.com

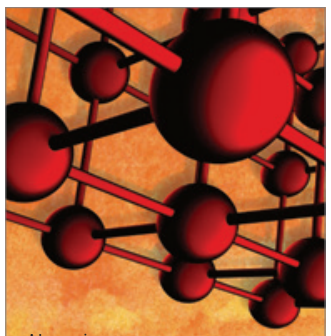

Materials Science and Engineering
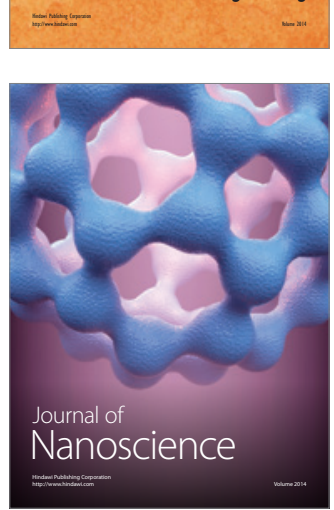
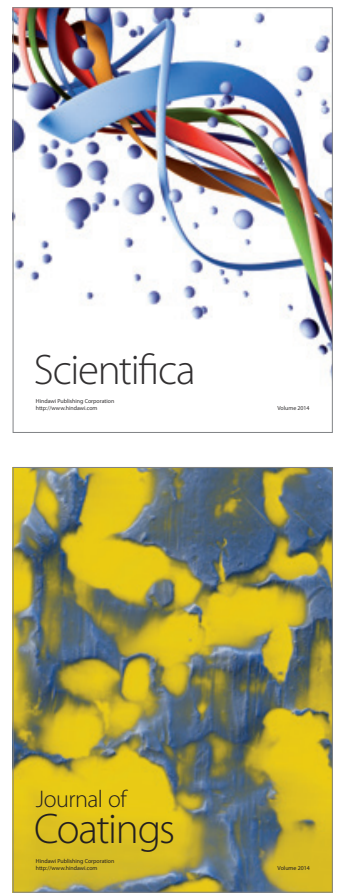
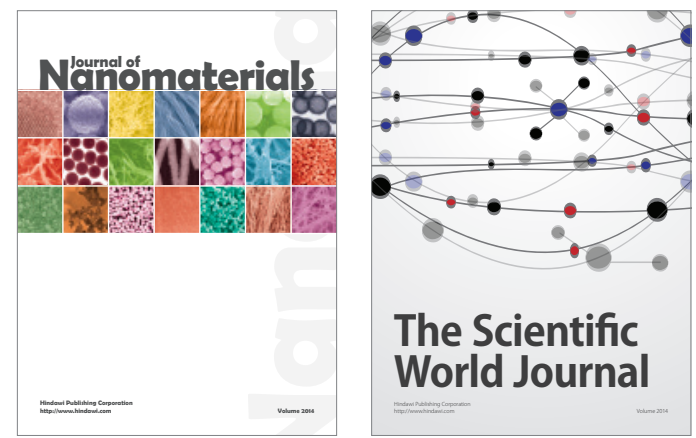

The Scientific World Journal
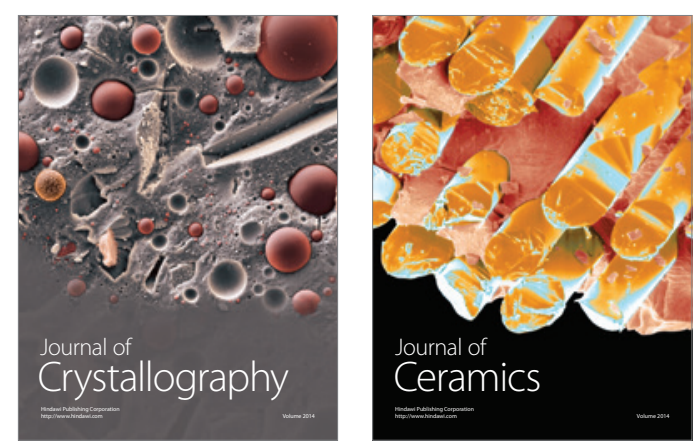
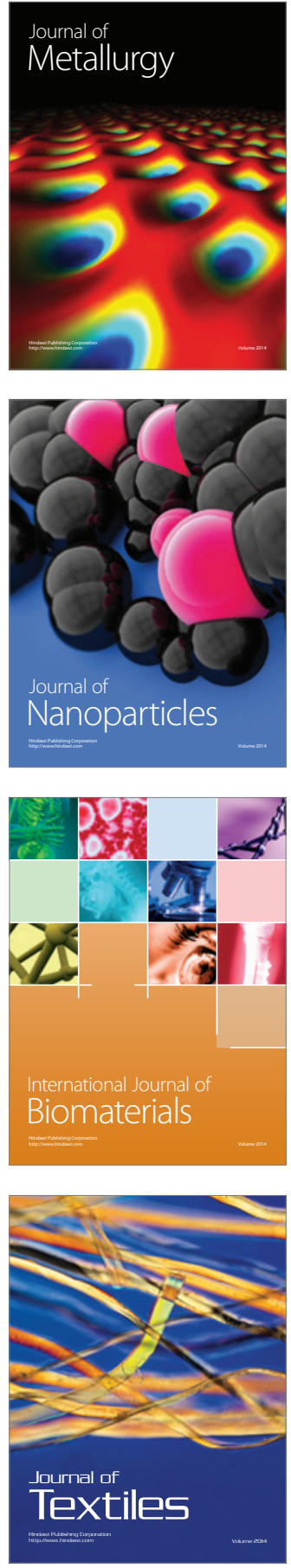